\title{
Preface
}

\section{PET-based Novel Imaging Techniques with Recently Introduced Radiotracers}

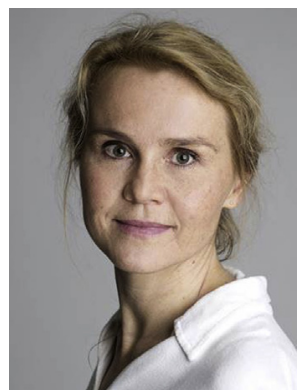

Mona-Elisabeth Revheim, MD, PhD, MHA

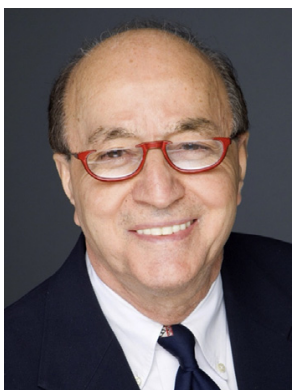

Abass Alavi, MD, MD (Hon), PhD (Hon), DSc (Hon)

Editors
Today, imaging research is an integral component of basic and clinical investigation in medicine. With the introduction of novel interventions, which are complex and systemic in nature, sophisticated imaging techniques along with new blood biomarkers, molecular histology, and advanced data analysis schemes have become a necessity in modern practice of medicine. These advances are allowing assessment of response at a molecular level with high precision and accuracy. In particular, molecular imaging modalities provide noninvasive and quantitative measures of the in vivo disease activity at the focal and global levels, which are well suited for longitudinal studies. These approaches are based on targeting the intended sites by well-characterized mechanisms that have been identified and characterized over the past decades. These exciting developments have been well illustrated by the articles in the current issue of PET Clinics, which includes 12 reviews covering a broad spectrum of relevant topics in the field of molecular imaging. We have made an effort to include scientific communications that describe clinical and research applications of novel PET tracers that are currently of great interest to the medical community.

Oncology still is one of the most active domains for applications of PET tracers, and therefore, 6 reviews in this issue have been dedicated to cancerrelated molecular imaging techniques. Hernes and colleagues and Seierstad and colleagues present systematic reviews of the clinical role of PET imaging with prostate-specific membrane antigen and fluciclovine (Axumin) in this cancer, respectively. With the advent of new receptor-based tracers in the assessment of hormonally active malignancies, the focus has now been shifted to some extent from fluorodeoxyglucose (FDG), since these compounds are more specific in nature. However, since the availability of such receptor-based tracers is limited worldwide, Yadav and colleagues discuss the strengths and limitations of various imaging techniques and point out that, despite recent development, we can still harness useful information from FDG imaging by focusing on breast, thyroid, neuroendocrine, and prostate malignancies. FDG-PET is particularly very sensitive in detecting undifferentiated cancer cells, which frequently occur in such malignancies and cannot be visualized by the specific tracers.

In recent years, new PET compounds have been synthesized by employing ${ }^{68} \mathrm{Ga}$ instead of ${ }^{18} \mathrm{~F}$ or ${ }^{11} \mathrm{C}$, and many of these tracers have been tested successfully and validated for examining several serious diseases and disorders. Positron emitting radioisotopes with long half-lives, such as ${ }^{64} \mathrm{Cu}$, have also gained recognition for certain applications for clinical and research purposes. In this issue, Johnbeck and colleagues describe the potential role of somatostatin-binding compounds that are labeled by both ${ }^{68} \mathrm{Ga}$ and ${ }^{64} \mathrm{Cu}$ for imaging neuroendocrine neoplasms. Insulinomas, neuroendocrine tumors with insufficient expression of somatostatin receptors $(<60 \%)$, are poor 
candidates for this approach. Therefore, a glucagon-like peptide-1 (GLP-1) receptor-based PET imaging agent, like radiolabeled exendin-4, has been introduced for targeting pancreatic $\beta$ cells in patients with hyperinsulinism. An article by Luo and colleagues provides an introduction to the development, clinical research, and perspective of GLP-1 receptor imaging and its high sensitivity and specificity in localizing insulinomas.

The introduction of novel PET tracers to image and assess central nervous system disorders has met serious challenges due to the PET tracers' ability to cross the blood-brain barrier and their specificity for the intended targets. Because of the nonspecificity of structural imaging techniques, there is a dire need for imaging brain tumors with tracers that allow assessing tumor response and the course of the disease. The role of PET tracers that are currently used to characterize brain tumors is discussed in a critical review by Borja and colleagues.

There has also been an emerging interest in understanding the role of the neuroimmune system in the pathophysiology of neurodegenerative and psychiatric diseases, and several PET tracers have been investigated with somewhat divergent and confusing results. Narayanaswami and colleagues summarize the findings from imaging with different generations of translocator protein $18-\mathrm{kDa}$ tracers (primarily microglia) and monoamine oxidase- $B$ radiotracers (astrocytes preferred). They also present results from a preclinical study in a lipopolysaccharide rat model of neuroinflammation. Another focus of investigation in molecular neuroimaging dealt with the protein depositions in head injuries and movement disorders. In the review by Ayubcha and colleagues, the state of tau radiotracer developments and the potential clinical role of tau-PET imaging are described in this domain. Furthermore, Bohnen and Frey evaluate possible targeting of protein depositions that are specific to neurodegenerative parkinsonism disorders. These include aggregates of misfolded tau proteins characteristic of progressive supranuclear palsy and $\alpha$-synuclein in Parkinson disease and multiple system atrophy.

Two articles are devoted to PET imaging of the cardiovascular system with different perspectives.
Hancin and colleagues describe a summary of non-FDG/NaF radiotracers that have been proposed for the diagnosis and management of cardiovascular disorders. The review by Joseph and colleagues deals with imaging of cardiac amyloidosis by both planar/single-photon emission computed tomography and PET/computed tomography (CT) and describes the current state of these modalities as they relate to this serious disease. The final article is a review of the role of $\mathrm{NaF}$ $\mathrm{PET} / \mathrm{CT}$ in detecting and characterizing musculoskeletal disorders with an emphasis on traumatic injuries, joint diseases, back pain, orthopedic complications, and metabolic bone disorders.

By now, it has become quite clear that among the existing modalities, molecular imaging with PET has the greatest prospects for becoming the examination of choice for personalized medicine in the foreseeable future. During the past 4 decades, many novel radiotracers have been introduced for PET imaging and have been shown to be of great value in certain settings. However, we must emphasize that FDG as a single tracer has brought about a revolution to medical imaging, and without its substantial impact, PET could not have survived as a sustainable discipline. As such, we salute our distinguished colleague, Henry Wagner, for labeling FDG as the "Molecule of the 20th Century."

Mona-Elisabeth Revheim, MD, PhD, MHA Division of Radiology and

Nuclear Medicine

Oslo University Hospital

Sognsvannsveien 20

0372 Oslo, Norway

Abass Alavi, MD, MD (Hon), PhD (Hon), DSc

(Hon)

Division of Nuclear Medicine

Department of Radiology

University of Pennsylvania

School of Medicine, Hospital of the

University of Pennsylvania

3400 Spruce Street

Philadelphia, PA 19104, USA

E-mail addresses:

monar@ous-hf.no (M.-E. Revheim)

Abass.Alavi@pennmedicine.upenn.edu (A. Alavi) 